\title{
EDITORIAL
}

\section{Ruminations on a new journal}

\author{
Grant Innes, MD
}

The Canadian Journal of
Emergency Medicine? ... What? Eh? ... Last December the CAEP Board voted unanimously to make the leap from newsletter to journal. After doing so, they were forced to choose an editor, which is a decision not to be taken lightly. A Board subcommittee deliberated for weeks before offering me the position. It was a tough decision, I'm told, but it would have been tougher had anyone else been willing to take the job.

My first task as editor was to determine why, exactly, we needed a new journal. The CAEP Board had plenty of reasons, but a good editor considers the needs of his or her readers, so I contacted all three of them.

One said, "Grant, Canada is a big country with a lot of diverse emergency physicians. It's time we had our own academic EM journal."

The second said, "I like the relaxed attitude and humour of Communiqué. Don't change a thing."

And the third said, "CJEM should be a forum for Canadian emergency physicians, Canadian research and Canadian issues."

Given this input, we decided that CJEM should be a truly Canadian journal: polite and humble, relaxed and humorous, yet highly scientific - a journal that is just as comfortable in the bathroom as in the recycling bin; a journal that publishes Canadian issues,

\section{Editor, CJEM}

such as overcrowded hospitals, curling injuries and hypothermia; a journal that tackles controversial matters head-on, such as Why do we spell "humour" with a " $u$ "? and Why is Canada the only country whose élite athletes believe marijuana is a performanceenhancing drug?

The CJEM editorship is a wonderful job - everything a person could want, apart from a source of taxable income — but it's more than just power lunches and cocktail parties. There are challenges galore. The first thing you notice when you become the editor of a new journal is that you have no articles to publish. This is good. It allows you to determine, without undue influence, the direction the journal should take. The second thing you notice when you become the editor of a major national publication is that you must interact with people from other parts of the country. This is easier said than done. Canada is a large country with inconvenient regions known as "time zones." Time zones are a fascinating phenomena that plague editors the world over.

I have tried to explain the scientific principles behind time zones on many occasions. However, it is a complex subject, difficult for the common mind to grasp so, using a hypothetical person from Toronto, I offer this illustration.

Every morning, at 8 o'clock sharp, our perplexed but well-meaning Torontonian pours his morning coffee and rubs his chin thoughtfully, pondering a question that only a journal editor could answer — a question like: "What colour ink will the new journal use? Will it be black like other journals?" Wishing to clarify this or another weighty issue, he calls me in Vancouver. Inexplicably, through the miracle of time zones, his call reaches my bedside telephone THREE HOURS before he even dialled. That's right! Amazingly, my phone rings at 05:00 in the morning. While this sounds unpleasant, it is actually good because, otherwise, my family would sleep through a wonderful sunrise and I would waste hours drooling on my pillow when I could be busily rejecting peoples' articles for no reason whatsoever. After clarifying the ink issue, our hypothetical Torontonian returns to his work, still rubbing his chin, wondering why West-Coasters are so damn snarky.

Having dealt with major problems like "no articles" and "time zones" it comes as a shock to the new editor's system when he realizes he must focus not on science and literature but, rather, on marketing and circulation. The first key strategy our consultants identified was for us to choose a catchy title that would jump out and grab the potential reader. We considered the Canadian Journal of Losing Weight and Having Better Sex and the Canadian Journal of Mostly-Naked Actors and Celebrities (our thanks to Dr. Bruce Campana for this suggestion) before we settled on the Canadian Journal of Emergency Medicine.

Continued on page 16 


\title{
ÉDITORIAL
}

\section{Méditations sur un nouveau journal}

\author{
Grant Innes, MD
}

$\mathrm{L}$ e Journal canadien de médecine d'urgence? ... Hein? Comment? ... En décembre dernier, le Conseil d'administration de l'ACMU votait à l'unanimité pour que le bulletin d'information devienne un journal. Par la suite, il dut choisir un rédacteur, décision à ne pas prendre à la légère. Un sous-comité du Conseil délibéra pendant des semaines avant de m'offrir le poste. La décision n'était pas facile, m'a-t-on dit, mais elle aurait été encore plus difficile si quelqu'un d'autre avait été prêt à accepter le poste.

Ma première tâche en tant que rédacteur était de déterminer pourquoi exactement, il nous fallait un nouveau journal. Le Conseil d'administration avait bon nombre de raisons, mais un bon rédacteur doit considérer les besoins de ses lecteurs; je les ai donc appelés tous les trois.

Un lecteur a dit : «Grant, le Canada est un grand pays avec beaucoup de médecins d'urgence. Il est temps que nous ayons notre propre journal académique de MU.»

Le deuxième m'a confié : «J'aime l'attitude décontractée et l'humour du Communiqué. Ne change rien.»

Et le troisième a déclaré : «Le $J C M U$ devrait être un forum pour les médecins d'urgence canadiens, la recherche canadienne et les préoccupations canadiennes.»

À partir de ces commentaires, nous avons décidé que le $J C M U$ devrait être

Rédacteur, JCMU un journal véritablement canadien. Il doit être poli et humble, décontracté et humoristique, mais hautement scientifique; un journal qui soit tout aussi confortable dans la salle de bain que dans le bac de recyclage; un journal qui parle des questions canadiennes, comme l'engorgement des hôpitaux, les blessures de curling et l'hypothermie; un journal qui attaque de front les questions controversées comme Pourquoi épelle-t-on «oedème» avec un «o»? et Pourquoi le Canada est-il le seul pays où les athlètes d'élite croient que la marijuana est une drogue qui améliore la performance?

Pour moi, le fait d'être nommé rédacteur du JCMU constitue le point culminant du rêve de toute une vie (devenir un demi-dieu) mais, bien que ce travail représente tout ce qu'une personne pourrait souhaiter, à part une source de revenu imposable, il ne se compose pas que de dîners d'affaires et de cocktails. Il y a des défis à la pelle.La première chose que vous constatez lorsque vous devenez rédacteur d'un nouveau journal, c'est qu'il n'y aucun article à publier. La deuxième chose que vous constatez lorsque vous devenez rédacteur d'une publication nationale importante, c'est que vous devez collaborer avec des gens de partout au pays. Voilà qui est plus facile à dire qu'à faire. Le Canada est un grand pays doté de régions fort embarrassantes mieux connues sous le nom de «fuseaux horaires.» Les fuseaux horaires constituent un phénomène scientifique fascinant qui tourmente les rédacteurs à travers le monde.

J'ai tenté d'expliquer les principes scientifiques qui sous-tendent les fuseaux horaires en plusieurs occasions. Cependant, le sujet est complexe et difficile à comprendre pour le commun des mortels. Permettez-moi d'illustrer mon propos à l'aide d'une personne fictive résidant à Toronto.

Chaque matin, à huit heures tapant, notre résidant de Toronto, perplexe mais bien intentionné, se verse son café matinal en se frottant pensivement le menton, réfléchissant à une question à laquelle seul un rédacteur de journal pourrait répondre: «Quelle couleur d'encre le nouveau journal utilisera-til? Sera-t-elle noire comme pour les autres journaux?» Anxieux de clarifier cette question importante ou toute autre du même ordre auxquelles nous, rédacteurs de journal, sommes confrontés quotidiennement, il m'appelle à Vancouver. Curieusement, par le miracle des fuseaux horaires, son appel atteint le téléphone sur ma table de chevet TROIS HEURES avant même qu'il n'ait signalé. En effet, surprenamment, mon téléphone sonne à 5 heures du matin. Bien que cette situation puisse vous sembler déplaisante, c'est en fait excellent. Sinon, ma famille manquerait un merveilleux lever de soleil et je perdrais de précieuses heures à baver sur mon oreiller alors que je pourrais m'occuper activement à rejeter pour aucune bonne raison les

suite à la page 16 


\section{Ruminations on a new journal}

Which leads me to another issue. Normally I wouldn't clutter up a perfectly good editorial with facts, but the following real-life situation is so humorous I had to include it, even though it's true. The amusing facts are as follows. After we performed a title search and confirmed that no one else publishes a Canadian Journal of Emergency Medicine, we successfully registered the name. Then, for reasons I can't recall at the moment, we consulted our lawyers, who determined, by conferring in expensive restaurants, that we could not use that title.
Why? you ask. Decades ago, someone trademarked the name "Emergency Medicine."

To the legally untrained mind this doesn't seem like a problem because Emergency Medicine is not the title we chose. However, our lawyers explained that "Canadian" and "Journal" cannot be trademarked, so when lawyers read "Canadian Journal of Emergency Medicine" they believe they are reading "Emergency Medicine" and this allows them to sue us. The bottom line is that any title including the words "Emergency Medicine" is off limits.
It's not clear how our situation differs from Annals of Emergency Medicine, the Journal of Emergency Medicine, the Australasian Journal of Emergency Medicine, and a dozen others. The only obvious difference is that these journals do not publish in Canada, but perhaps there is another, correctable explanation. If not, rest assured that I will publish the Canadian Journal of Emergency Medicine from wherever I have to - even if it means moving the editorial office to Bermuda or Tahiti.

By which time our Toronto colleagues will have sorted out the conundrum of time zones or I'll have a real office number.

\section{Méditations sur un nouveau journal}

articles qu'on m'a envoyés. Après avoir clarifié la question de l'encre, notre résidant de Toronto hypothétique retourne au travail, se frottant toujours le menton en se demandant pourquoi donc les gens de l'Ouest sont si râleurs.

Ayant réglé les problèmes majeurs comme «pas d'articles» et les «fuseaux horaires», le nouveau rédacteur est surpris de constater qu'il doit se concentrer non pas sur la science et la littérature mais plutôt sur le marketing et la circulation. La première stratégie clé identifiée par nos consultants était de trouver un titre accrocheur qui attirerait immédiatement l'attention du lecteur éventuel. Le conseil de rédaction réfléchit longuement à cette question. Nous avons considéré le Journal canadien pour perdre du poids et améliorer sa vie sexuelle et le Journal canadien d'acteurs et de célébrités à moitié nus (merci au $\mathrm{D}^{\mathrm{r}}$ Bruce Campana pour cette suggestion) avant de choisir Canadian Journal of Emergency Medicine (Journal canadien de médecine d'urgence).
Voilà qui m'amène à une autre question. Normalement, je n'alourdirais pas un éditorial parfaitement équilibré avec des faits, mais la situation véridique que je vais vous décrire est tellement drôle que je me devais de la partager avec vous. Voici donc ce qui s'est produit : Après avoir effectué des recherches pour confirmer qu'aucune autre publication ne portait le nom de Canadian Journal of Emergency Medicine, nous avons enregistré ce nom sans problèmes. Puis, pour des raisons que j'oublie maintenant, nous avons consulté plusieurs avocats fort coûteux. Ils ont déterminé, lors de longues discussions dans de grands restaurants, que nous ne pouvions pas utiliser ce titre. Il semble que, il y a des dizaines d'années, quelqu'un ait fait de l'expression «emergency medicine» une marque déposée.

Pour le commun des mortels, cela ne semble pas poser problème, puisque «emergency medicine» n'est pas le titre que nous avons choisi. Or, nos avocats nous ont expliqué que «Canadian» et «Journal» ne pouvaient être déposés. Donc, lorsque des avocats lisent «Canadian Journal of Emergency Medicine», ils croient lire «Emergency Medicine», ce qui leur donne le droit de nous poursuivre. En fin de compte, tout titre qui comprend les mots «Emergency Medicine» est hors limites.

Nous ne voyons pas comment notre situation est différente des Annals of Emergency Medicine, du Journal of Emergency Medicine, du Australasian Journal of Emergency Medicine, et d'une douzaine d'autres publications. La seule différence notable est que ces journaux ne sont pas publiés au Canada, mais peut-être trouveronsnous une autre explication. Sinon, soyez assurés que je me sacrifierai pour vous. Si nécessaire, je publierai le Canadian Journal of Emergency Medicine à partir des Bermudes ou de Tahiti, là où il nous sera permis d'utiliser notre véritable nom spirituel.

À ce moment-là, peut-être que nos collègues de Toronto auront compris la complexité des fuseaux horaires ou que j'aurai un vrai numéro de bureau. 